\title{
HPV prevalence and concordance in the cervix and oral cavity of pregnant women
}

\author{
E.M. Smith ${ }^{1,2}$, J.M. Ritchie ${ }^{3}$, J. Yankowitz ${ }^{2}$, D. Wang ${ }^{1,4}$, L.P. Turek ${ }^{4,5}$ and \\ T.H. Haugen ${ }^{4,5}$ \\ ${ }^{1}$ Department of Epidemiology, College of Public Health, University of Iowa, IA \\ ${ }^{2}$ Department of Obstetrics/Gynecology, College of Medicine, University of Iowa, IA \\ ${ }^{3}$ Department of Biostatistics, College of Public Health, University of Iowa, IA \\ ${ }^{4}$ Veterans Affairs Medical Center, Iowa City, IA \\ ${ }^{5}$ Department of Pathology, College of Medicine, University of Iowa, IA
}

Objectives: This investigation examined human papillomavirus (HPV) in pregnant women in order to characterize viral prevalence, types and concordance between infection in the cervix and in the oral cavity. Methods: A total of 577 pregnant women seeking routine obstetric care were evaluated for HPV infection in their cervix during gestation and immediately before delivery, and in the oral cavity during gestation. Male partners present during the gestational clinic visit also provided a specimen from their oral cavity. HPV assessment was performed by PCR, dot blot hybridization and DNA sequencing. A sexual and health questionnaire was completed by the pregnant women.

Results: HPV prevalence in women was $29 \%$ in the cervix and $2.4 \%$ in the oral cavity. Among those with both gestational and delivery specimens, $35 \%$ were infected at least once and $20 \%$ had infection at both intervals. At delivery, $68 \%$ of infected women had an oncogenic HPV type in the cervix. There was no type-specific HPV concordance between the two cervical specimens, nor cervical and oral results in women, nor with cervical and oral findings between partners.

Conclusion: The lack of association in HPV positivity and types between the cervix and oral cavity in these women suggests that self-inoculation is uncommon. This source of infection does not appear to be from oral contact with a current male partner, since there also was no concordance between partners. These results suggest either other modes of HPV transmission or differences in susceptibility to HPV infection or its clearance in the oral cavity and genital mucosa.

Key words: HPV; Pregnancy; Oral Cavity; Genital; Concordance; Persistence

Human papillomavirus (HPV) in the genital area is thought to be transmitted primarily through direct mucosal contact and is causal in the development of cervical cancer, in a larger proportion of other carcinomas in the lower genital tract, and in benign lesions ${ }^{1}$. Persistent infection with oncogenic, high-risk (HR) HPV types is particularly associated with development of these preneoplastic and malignant lesions ${ }^{2,3}$ and evidence suggests that progression of cervical lesions is almost always associated with persistence of HPV-HR types ${ }^{2}$.

Correspondence to: Elaine M. Smith, Department of Epidemiology, College of Public Health, University of Iowa, Iowa City, IA 52242, USA. Email: elaine-smith@uiowa.edu 
HPV infection also may be transmitted vertically during parturition ${ }^{4-6}$. This proposition is of concern since the prevalence of detectable maternal HPV infection during pregnancy reportedly may rise during the second and third trimester above that of non-pregnant women, thus increasing the probability of HPV exposure to perinatal transmission at the time of delivery ${ }^{5,7}$. There is evidence that the same viral types found in cervical carcinoma are detected in and risks for $20-25 \%$ of head and neck cancers as well ${ }^{8,9}$. These include oncogenic mucosal types HPV-16, 18,31 , and $33^{8,9}$. This consistency in viral types in the different mucosal areas would further suggest sexual transmission of HPV. Kellokoski et al. ${ }^{10}$, in one of the few published studies to evaluate whether HPV types were similar in the oral and genital sites of women, found only $3 \%$ had HPV in their oral cavity. Concordance was less than $1 \%$ despite the fact that the population had a history of HPV genital lesions. To date, it is still not clear whether pregnancy is associated with an increased prevalence of HPV detected during gestation, nor whether the prevalence and types of HPV are the same in the oral cavity as in the cervix generally.

The purpose of this study was to describe the prevalence and types of HPV in the cervix and the in oral cavity of pregnancy women and to evaluate concordance between these two sites. The findings will clarify our knowledge about infection rates in pregnant women compared with what is known about young non-pregnant women. In addition, this will be one of the first large studies of prevalence and concordance rates between genital and oral sites, and of possible transmission routes between sexual partners.

\section{METHODS}

\section{Study population}

A total of 582 pregnant women who were being seen for their routine obstetric examinations were successfully recruited into the study between September 1997 and November 2000 at the Department of Obstetrics and Gynecology of the University of Iowa medical college. Participants gave informed consent to an approved university human subjects consent form. The number of women who refused was $83 ; 16$ were too ill or had complicated pregnancies; 11 had a language barrier; 2 were mentally unable to consent to participate; and 13 were not going to deliver at the research hospital site and thus would not be available for a cervical assessment of HPV at the time of delivery.

\section{Data collection}

Questions about sociodemographics, tobacco and alcohol usage, contraceptive practices, reproductive and sexual history and other risks of genital intra-epithelial neoplasia (SIL), cancer, and condylomata acuminata were asked at enrollment. Patients were asked about previous history of HPV-related genital diseases: cervical, vulvar, or vaginal SIL or cancer, and genital warts. A medical form was completed using the patient's medical chart, laboratory tests and physician clinical evaluations, and included current and historical data regarding abnormal Pap smear diagnoses and previous history of HPV-related genital conditions and treatments.

\section{Pap smear and HPV specimen collection}

Pap smears were collected to screen for current cervical cytologic abnormalities and were followed by a genital specimen to detect HPV DNA. A pap smear was performed first and results were based on the Bethesda system ${ }^{11}$. The cervical HPV DNA specimen was collected with an $20 \mathrm{~cm}$ cotton tipped swab dipped in $5 \mathrm{ml}$ normal saline and inserted in the vagina. The cervix, the ectocervix and pooled secretions in the posterior vaginal fornix were swabbed using a circular motion to loosen squamous cells. The swab was placed back into the tube of normal saline and agitated in a circular motion to loosen squamous cells, and was discarded before the tube was capped. The second HPV DNA maternal specimen was collected immediately before delivery, regardless of whether the woman had a vaginal or cesarean delivery. Specimens were immediately refrigerated. A cell count was performed using a hemocytometer and recorded for use in the laboratory procedures to assure an 
adequate number of nucleated squamous cells in the PCR reaction. Specimens were subsequently frozen at $-80^{\circ} \mathrm{C}$ until viral testing. The two maternal cervical samples were collected by the attending physician at 35 weeks' gestation, on average, and again during labor immediately before delivery (at about 39 weeks).

Women with HPV infection detected at both time periods were described as having persistent infection, whereas those with HPV detected at only one of the two time periods were said to have non-persistent infection. The oral rinse specimens were collected during the third trimester clinic visit or, if women attended the clinic only when in labor, it was collected at that time. A total of 69 male partners who accompanied women at either of these times were asked to participate and to submit an oral specimen for HPV testing so that viral concordance could be examined. Patients were asked to rinse the mouth vigorously with $5 \mathrm{ml}$ normal saline for $30 \mathrm{~s}$ and expectorate into a pill cup which was then placed in to a $10 \mathrm{ml}$ tube and refrigerated. A cell count and freezing were performed as described for the cervical specimens. Because of inadequate oral cell count 1 man and 2 women were excluded, and another 3 women were excluded because the oral specimen was collected some time after delivery, leaving 577 women and 68 current male partners in the study.

\section{HPV DNA assessment}

Procedures for sample preparation, PCR analyses and DNA hybridization were described previously $^{12}$. In the DNA processing of the specimens, repeat HPV-negative oral cells were included as a control for contamination. All processing of samples was performed in a building separate from and before going to the research laboratory for the day. The DNA extraction was followed by the PCR reaction which included positive controls containing $0.1 \mathrm{pg}$ and $1.0 \mathrm{pg}$ of HPV plasmid DNA with the primers. Negative controls were $0.025 \mu \mathrm{g}$ of human DNA from oral exfoliated squamous cells, normal saline and PCR buffer. Amplification with primers specific for $\beta$ globin was used to confirm the presence of adequate cellular DNA in each specimen for HPV
DNA testing ${ }^{13}$. Specimens repeatedly negative for the $\beta$-globin fragment were excluded from the analyses. No maternal or paternal specimens were judged inadequate on the basis of the $\beta$-globin result.

The primers used to identify HPV in the samples, MY09 and MY11 ${ }^{14}$, are a mix of primers amplifying a portion of the L1 gene of HPV types. A primer to improve amplification of HPV-51 also was included ${ }^{3}$. Initially, amplified samples were electrophoresed through agarose gels with $10^{-3} \mathrm{ml}$ of the amplification products to detect HPV DNA. If specimens were band-negative, they were hybridized by dot blot with the inclusion of the GP5 + primer in a hemi-nested fashion to determine which samples would require re-amplification for DNA sequencing ${ }^{15}$. Each sample prepared for initial PCR amplification contained 30000 nucleated cells. The dot blot detected less than $10 \mathrm{SiHa}$ cells in a spiked sample, or less than 1 copy of HPV/1000 squamous cells in sample specimens. When discordant HPV types were found in separate samples from the pregnant women, we performed additional PCRs with type-specific primers which amplify portions of the E6 ORF. This allowed additional HPV types to be detected specifically in the presence of one or more other types. E6 type-specific primers pairs used in this study were: CAGGACCCACAGGAGCGACC and ATCGACCGGTCCACCG ACCC amplifying nt 110-509 of HPV-16, TGCCAGACCTGTGCACAACG and GCACC GTCGACACTGTCCTG amplifying nt 147-510 of HPV-39, GGGAAACACCACGAACGCTG and GCGCATTGCCCCGTCCAACG amplifying nt 113-506 of HPV-51, and GCAAGGACTA CGAGGTGGAC and TATGCCTGTACCTGA TGCTC amplifying nt 139-457 of HPV-61.

HPV types were determined using DNA sequencing of amplified HPV DNA evaluated at the DNA Core at the College of Medicine facilities. This was performed by dye-termination on a DNA sequencer (Applied Biosystems-PE Biosystems Group, Foster City, CA) and nucleotide sequences were compared with GenBank NCBI, NIH sequences using the BLAST program $^{16}$. HPV types were defined as either highrisk (HR) or low-risk (LR) on the basis of types that have been associated with cervical SIL, 
cancer or benign lesions such as genital warts. Specific HPV types were identified if there was a known type that matched by $>90 \%$. The HPV-HR types detected in this study were: 16,18,31,33,35,39,51,54,58,59,68 and 70. HPV-LR types identified included: $6,11,38,44,53,56,61,66,69,83$ and five unnamed types (AF091451, AJ010822, L38388, U12480, U12489, U12490). The unnamed types were all related to benign and low-risk cutaneous HPV types when a sequence comparison with GenBank was performed. Other samples were defined as "positive unclassified" when they were identified as HPV based on the size of the band by Southern blot but had insufficient DNA for typing. There were no cervical and only $0.3 \%$ of oral samples in this category.

\section{Data analysis}

Logistic regression was used to examine the association between HPV detection and potential risk factors and to examine the association between ever HPV-related disease history or ever abnormal Pap smear and persistent HPV groupings while adjusting for other risk factors. HPV detection outcomes included ever HPV positivity, persistent HPV positivity, and persistent HPV-HR in cervix samples and HPV positivity in oral rinse samples. Adjusted odds ratios (ORs) were based on multiple logistic regression models determined by a backward stepwise selection procedure with significance set to $p<0.05$. Variables considered in the modeling process were: age, education, age at first intercourse, number of partners, number of pregnancies and tobacco status. Ever HPV-related disease history and ever abnormal Pap smear result were included in the modeling process for HPV detection only. Confidence intervals (95\% CI) for ORs were calculated using the standard errors from the corresponding logistic regression models and the normal approximation. Due to low positivity rates, exact logistic regression was used to examine the association between HPV detected in oral samples and potential risk factors at that site. Pearson's correlation coefficient was used to examine the association between two quantitative variables. To determine if there was a significant difference in the HPV rates between the two cervical specimen collections (gestation and delivery), McNemar's test was used. All analyses were performed using the SAS statistical package ${ }^{17}$.

\section{RESULTS}

Table 1 shows the demographic characteristics and potential risk factors of HPV in the cervix of 577 pregnant women comparing the 165 ever HPVinfected women to the $412 \mathrm{HPV}$-negative defined as negative in the cervix at the time interval tested, during pregnancy (C1) or labor (C2). The table also includes separate analyses for the 60 women with persistent HPV infection, defined as HPV + in both $\mathrm{C} 1$ and $\mathrm{C} 2$ specimens, compared with the 199 women who were HPV-negative at both of these intervals. Controlling for age, number of partners, smoking status, and history of HPV disease, the overall risk of HPV detection was greater in the youngest, unmarried, less educated, those with $\geqslant 3$ sex partners, and current cigarette users. There was no significant association with parity or duration of oral contraceptive use (data not shown). Those with a history of cervical SIL and cancer had an elevated risk in the ever HPV + group, whereas those with a current diagnosis of ASCUS or L-SIL did not.

Those with persistent HPV infection had a risk history similar to the HPV + women overall (Table 1), as did women with HPV-HR persistent infection (data not shown). There were, however, notable differences in the lack of association with age and smoking but a much higher risk with $\geqslant 3$ sex partners in the persistently infected. The 46 women with HPV detected in their cervix at only one of the two time periods, a non-persistently infected group, had risks similar to those for the $\mathrm{HPV}+$ women overall (data not shown). The adjusted age-related risk was between that of the ever $\mathrm{HPV}+$ and the persistently $\mathrm{HPV}+$ risk $(\leqslant 24$ vs $\geqslant 33$ years; adj. $\mathrm{OR}=2.6,1.1-6.5)$.

\section{Genital HPV prevalence and types}

The cumulative HPV prevalence was $29 \%$ in the cervix (Table 2). The most frequently reported HR types were HPV-16, HPV-31, HPV-18, and HPV-51; and the most common LR types included HPV-53 and HPV-83. Among the 450 
Table I Baseline demographic characteristics and risk factors for HPV in the cervix of pregnant women $(n=577)$

\begin{tabular}{|c|c|c|c|c|c|c|c|c|}
\hline \multirow[b]{2}{*}{ Characteristic } & \multicolumn{4}{|c|}{ All HPV + and HPV- women $(n=577)$} & \multicolumn{4}{|c|}{ Persistent HPV + and HPV- women $(n=259)$} \\
\hline & $n$ & $\% H P V$ & Adj. $O R^{4}$ & $95 \% \mathrm{Cl}$ & $n$ & $\% H P V$ & Adj. $O R^{5}$ & $95 \% \mathrm{Cl}$ \\
\hline \multicolumn{9}{|l|}{$\mathrm{Age}^{3}$} \\
\hline$\leqslant 24$ & 140 & 47.1 & 4.0 & $2.4-6.7$ & 58 & 37.9 & $\mathrm{I} .7$ & $0.7-4.4$ \\
\hline $25-29$ & 223 & 25.1 & 1.6 & $1.0-2.6$ & III & 18.9 & 1.3 & $0.6-2.9$ \\
\hline$\geqslant 30$ & 214 & 20.1 & 1.0 & - & 90 & 18.9 & 1.0 & - \\
\hline \multicolumn{9}{|l|}{ Marital status } \\
\hline married & 443 & 21.9 & 1.0 & - & 186 & 14.0 & I.0 & - \\
\hline never married/div/sep & 134 & 50.8 & 1.8 & I.I-3.0 & 73 & 46.6 & 2.4 & I.I-5.0 \\
\hline \multicolumn{9}{|l|}{ Education $^{3}$} \\
\hline$\leqslant 12$ & 132 & 47.0 & 2.0 & $1.0-3.8$ & 68 & 44.1 & 7.0 & $2.7-18.0$ \\
\hline $13-16$ & 254 & 28.7 & 1.5 & $0.9-2.6$ & 112 & 19.6 & 1.9 & $0.7-4.7$ \\
\hline$\geqslant 17$ & 191 & 15.7 & 1.0 & - & 79 & 10.1 & I.0 & - \\
\hline \multicolumn{9}{|l|}{ Age first intercourse ${ }^{3}$} \\
\hline$\leqslant 17$ & 305 & 36.7 & 0.9 & $0.4-1.7$ & 137 & 31.4 & 1.5 & $0.4-6.3$ \\
\hline $18-19$ & 136 & 25.7 & 0.9 & $0.5-1.9$ & 65 & 21.5 & 1.6 & $0.4-7.0$ \\
\hline$\geqslant 20$ & 136 & 13.2 & 1.0 & - & 57 & 5.3 & $\mathrm{I} .0$ & - \\
\hline \multicolumn{9}{|l|}{ Number of partners } \\
\hline $\mathrm{I}-2$ & 186 & 10.8 & 1.0 & - & 87 & 5.8 & I.0 & - \\
\hline $3-5$ & 195 & 35.4 & 3.5 & $2.0-6.3$ & 85 & 29.4 & 5.7 & $1.9-16.6$ \\
\hline$\geqslant 6$ & 190 & 39.0 & 3.3 & $1.8-6.2$ & 84 & 34.5 & 6.2 & $2.1-18.5$ \\
\hline \multicolumn{9}{|l|}{ Parity } \\
\hline I & 176 & 26.7 & 1.0 & - & 80 & 26.3 & 1.0 & - \\
\hline $2-3$ & 282 & 26.2 & 1.2 & $0.7-1.9$ & 134 & 15.7 & 0.6 & $0.3-1.2$ \\
\hline$\geqslant 4$ & 119 & 37.0 & 1.6 & $0.9-2.9$ & 45 & 40.0 & 1.2 & $0.5-2.9$ \\
\hline \multicolumn{9}{|l|}{ Smoking (pack-years) } \\
\hline Never & 375 & 21.3 & 1.0 & - & 165 & 14.6 & 1.0 & - \\
\hline Past & 122 & 33.6 & 1.1 & $0.6-1.8$ & 56 & 32.1 & 1.7 & $0.7-4.4$ \\
\hline Current & 80 & 55.0 & 2.8 & $1.5-5.0$ & 38 & 47.4 & 1.1 & $0.5-2.6$ \\
\hline \multicolumn{9}{|c|}{ HPV-related disease history } \\
\hline Never & 434 & 23.5 & 1.0 & - & 195 & 15.9 & 1.0 & - \\
\hline Condylomata & 64 & 39.1 & 1.5 & $0.9-2.9$ & 22 & 40.9 & 1.9 & $0.7-5.3$ \\
\hline Cervical SIL & 103 & 46.6 & 2.2 & I.4-3.7 & 51 & 47.1 & 3.3 & $1.5-6.8$ \\
\hline Cervical cancer & 7 & 71.4 & 5.1 & $0.9-30.5$ & 4 & 50.0 & $\mathrm{I} .7$ & $0.2-14.7$ \\
\hline \multicolumn{9}{|l|}{ Pap smear } \\
\hline WNL & 389 & 23.9 & 1.0 & - & 176 & 17.1 & 1.0 & - \\
\hline ASCUS & 117 & 35.0 & 1.2 & $0.7-2.1$ & 49 & 30.6 & 1.2 & $0.5-3.2$ \\
\hline L-SIL & 103 & 46.6 & 1.4 & $0.6-3.4$ & 51 & 47.1 & I.0 & $0.2-4.8$ \\
\hline
\end{tabular}

'Compared HPV + vs. HPV-; ${ }^{2} \mathrm{HPV}+$ at $\mathrm{Cl}$ and $\mathrm{C} 2$ vs. HPV- at $\mathrm{Cl}$ and $\mathrm{C} 2 ;{ }^{3}$ in years; ${ }^{4}$ adjusted for age, number of partners, smoking status and HPV-related disease history; ${ }^{5}$ adjusted for education, number of partners and HPV-related disease history, div, divorced; sep, separated

women with a C1 specimen, the HPV + rate was $26 \%$, and among the 432 women with a C2 specimen the rate was similar at $25 \%$. Based on either time period, the ever HPV-HR rate was $18 \%$ and the ever HPV-LR was 13\%. The prevalence of HPV decreased markedly with age: at $<25$ years it was $47 \%$, at $25-30$ years it was $25 \%$ and at $>30$ years it was $20 \%$. Those
$<25$ years old were at significantly greater risk of infection than those $\geqslant 25$ years old: $O R=2.3$, $1.4-3.6$

\section{HPV persistence and concordance}

Among the 305 women with both C1 and C2 specimens, 35\% were ever detected with HPV at 
Table 2 HPV prevalence and types in the cervix and oral cavity of pregnant women $(n=577)$

\begin{tabular}{|c|c|c|c|c|}
\hline \multirow[b]{2}{*}{ HPV status } & \multicolumn{2}{|c|}{ Cervix } & \multicolumn{2}{|c|}{ Oral cavity } \\
\hline & $n$ & $\%$ & $n$ & $\%$ \\
\hline $\mathrm{HPV}+{ }^{1,2}$ & 165 & 28.6 & 14 & 2.4 \\
\hline $\mathrm{HPV}-\mathrm{HR}^{2}$ & 104 & 18.0 & 9 & 1.6 \\
\hline 16 & 35 & 6.1 & 3 & 0.5 \\
\hline 18 & 15 & 2.6 & 0 & - \\
\hline 31 & 21 & 3.6 & 2 & 0.3 \\
\hline 33 & 4 & 0.7 & I & 0.2 \\
\hline 35 & I & 0.2 & 0 & - \\
\hline 39 & 2 & 0.3 & $\mathrm{I}$ & 0.2 \\
\hline 51 & 15 & 2.6 & 0 & - \\
\hline 54 & 4 & 0.7 & 0 & - \\
\hline 58 & 3 & 0.5 & 0 & - \\
\hline 59 & 2 & 0.3 & 0 & - \\
\hline 68 & 0 & - & I & 0.2 \\
\hline 70 & 6 & 1.0 & I & 0.2 \\
\hline HPV-LR ${ }^{2}$ & 73 & 12.7 & 5 & 0.9 \\
\hline 6 & 7 & 1.2 & I & 0.2 \\
\hline 11 & 3 & 0.5 & 0 & - \\
\hline 38 & I & 0.2 & 0 & - \\
\hline 44 & 1 & 0.2 & 0 & - \\
\hline 53 & II & 1.9 & 0 & - \\
\hline 56 & 2 & 0.3 & 0 & - \\
\hline 61 & 10 & 1.7 & 0 & - \\
\hline 66 & 5 & 0.9 & 0 & - \\
\hline 69 & I & 0.2 & 0 & - \\
\hline 83 & II & 1.9 & 0 & - \\
\hline$++^{5}$ & 0 & - & 2 & 0.3 \\
\hline AF09I45I & I & 0.2 & 0 & - \\
\hline AJ0I0822 & 12 & 2.1 & 0 & - \\
\hline L38388 & 0 & - & I & 0.2 \\
\hline UI2480 & 5 & 0.9 & I & 0.2 \\
\hline UI2489 & 1 & 0.2 & 0 & - \\
\hline UI2490 & 5 & 0.9 & 0 & - \\
\hline HPV persistence ${ }^{3}$ & 60 & 19.7 & - & - \\
\hline $\mathrm{HR}$ & 32 & 10.5 & - & - \\
\hline LR & 16 & 5.2 & - & - \\
\hline Concordance $^{4}$ & 40 & 13.1 & - & - \\
\hline
\end{tabular}

'2I with different types during interval among those with both $\mathrm{Cl}$ and C2 samples, a single oral specimen collected; ${ }^{2}$ ever positive at any time interval; ${ }^{3}$ among $n=305$ women with $\mathrm{Cl}$ and $\mathrm{C} 2$ sample; ${ }^{4}$ same type at Cl and C2: HPV-I6 (II), HPV-I8 (4), HPV-3I (5), HPV-33 (I), HPV-39 (2), HPV-5I (2), HPV-54 (I), HPV-70 (2), HPV-6 (I), HPV-II (I), HPV-53 (2), HPV-6I (I), HPV-83 (I), AJ0I 0822 (2), UI 2480 (2), UI 2490 (2); ${ }^{5} \mathrm{HPV}$ positive but could not be classified by type

one or both time points (Table 3). There was no significant difference in the positivity rate be- tween the gestational and delivery samples: $28 \%$ at $\mathrm{C} 1$ and $26 \%$ at $\mathrm{C} 2(p=0.4)$. When examined for prediction of HPV at the time of delivery based on the result at C1, 56\% (60/106) had persistent infection. The most common persistent HR types detected in the cervix were similar to those found overall: HPV-16, HPV-31 and HPV-18. The only LR type more frequently reported was HPV-53. There was no difference in the percentage between those who initially had a negative HPV test at C1 and then were HPV + at $\mathrm{C} 2$ compared with the percentage whose gestational test was positive and then changed to negative at delivery (9-11\%).

Because amplification with general primers may fail to detect HPV types when dual infections are present, type-specific PCR was performed focusing on women with discordant HPV types. In none of these cases was the discordance resolved by detection of additional HPV types. The type-specific concordance rate in the persistently infected women was $67 \% 40 / 60$ ), i.e. $47 \%$ HR, 20\% LR. Of those with HPV-HR types detected during the $\mathrm{C} 1$ pregnancy sampling, 67\% (32) remained $\mathrm{HR}$ with the $\mathrm{C} 2$ delivery testing and another 9\% (22) who were HPV-LR or negative at $\mathrm{C} 1$ subsequently converted to a $\mathrm{HR}$ type at C2 (Table 3). At the time of delivery, 68\% of those with an HPV infection had a high-risk type in the cervix.

\section{HPV persistence, HPV-related diseases and abnormal Pap smears}

Women with a history of an HPV-related disease had a higher frequency of infection. A previous history was lowest in the HPV-negative, higher in the non-persistent, and highest in the persistently infected group (Table 4). When compared with those who were HPV-negative for a history of an HPV cervical lesion at both C1 and C2, the adjusted ORs increased two to three times in those with non-persistent or persistent infection. The percentage having an abnormal Pap smear during the study period also increased from lowest in HPV-negative to highest in persistent infection, but only those with persistent infection had a significantly elevated risk with an abnormal Pap smear. When only high-risk HPV types were 
Table 3 HPV persistence and non-persistence in the cervix $(n=305)$

\begin{tabular}{lcccc}
\hline & \multicolumn{4}{c}{ HPV type at delivery } \\
\cline { 2 - 5 } HPV type at gestation & High-risk & Low-risk & Negative & Total \\
\hline High-risk & $32(10.5 \%)$ & $5(1.6 \%)$ & $11(3.6 \%)$ & $48(15.7 \%)$ \\
Low-risk & $7(2.3 \%)$ & $16(5.3 \%)$ & $15(4.9 \%)$ & $38(12.5 \%)$ \\
Negative & $15(4.9 \%)$ & $5(1.6 \%)$ & $199(65.3 \%)$ & $219(71.8 \%)$ \\
Total & $54(17.7 \%)$ & $26(8.5 \%)$ & $225(73.7 \%)$ & $305(100.0 \%)$ \\
\hline
\end{tabular}

Table 4 HPV-related history, abnormal Pap smear and HPV infection

\begin{tabular}{|c|c|c|c|c|c|c|c|c|c|c|}
\hline \multirow[b]{2}{*}{ Outcome } & \multicolumn{3}{|c|}{ HPV-negative $^{\prime}(n=198)$} & \multicolumn{3}{|c|}{ Non-persistent $(n=46)$} & \multicolumn{4}{|c|}{ Persistent $(n=60)$} \\
\hline & $\%$ & $n$ & $\%$ & $n$ & $O R$ & $95 \% \mathrm{Cl}$ & $\%$ & $n$ & OR & $95 \% \mathrm{Cl}$ \\
\hline HPV-related disease history ${ }^{2}$ & 17.2 & 34 & 34.8 & 16 & 2.1 & $0.98-4.4$ & 48.3 & 29 & 2.9 & $1.5-5.6$ \\
\hline \multirow[t]{2}{*}{ Abnormal Pap smear ${ }^{2}$} & 26.3 & 52 & 39.1 & 18 & 1.3 & $0.7-2.7$ & 50.0 & 30 & 2.0 & $1.0-3.7$ \\
\hline & & & & \multicolumn{3}{|c|}{ HR non-persistent $(n=38)$} & \multicolumn{4}{|c|}{ HR persistent $(n=32)$} \\
\hline HPV-related disease history ${ }^{2}$ & & & 47.4 & 18 & 3.2 & I.5-7.| & 46.9 & 15 & 2.4 & $1.0-5.6$ \\
\hline Abnormal Pap smear ${ }^{2}$ & & & 55.3 & 21 & 2.7 & I.3-5.6 & 50.0 & 16 & 1.8 & $0.8-4.1$ \\
\hline
\end{tabular}

'Missing Pap smear and HPV-related disease history for I woman; ${ }^{2}$ adjusted for number of partners

evaluated, both non-persistently and persistently infected groups had elevated adjusted risks.

\section{Oral-HPV results}

Among the 577 oral rinse specimens taken at the time of the cervical HPV collection, only $2.4 \%$ (14) of women were HPV + in the oral mucosa (Table 2). Almost all HPV types detected in the oral cavity were detected in the cervix. However, only $1 \%(6)$ had an infection in both the cervix and the oral cavity at the same time, but none had the same type detected in both mucosal areas. The majority of oral infections were HR types and 2 were detected in women with a HR cervical type: HPV-16 oral/HPV-58 cervix and HPV-68 oral/HPV-16 cervix. In contrast to genital HPV infections, the frequency and risk of detection in the oral cavity with HPV increased with age $(\mathrm{OR}=1.1 /$ year, $1.0-1.2)$ and parity $\geqslant 4$ $(\mathrm{OR}=4.0,1.2-13.7)$. Although not statistically significant, HPV-related risks associated with an increased odds or oral infection included a history of genital warts $(\mathrm{OR}=2.1)$ and cervical cancer $(\mathrm{OR}=7.0)$. A prominent oral and cervical cancer risk was elevated also in those infected in the oral cavity: smoking duration $\geqslant 5$ packyears $(\mathrm{OR}=3.8,1.1-12.8)$.

The HPV positivity rate in oral specimens collected from 68 current male partners who visited the clinic with the pregnant women was $5.9 \%$ (4). None of the infected men had female partners who concurrently were infected in either the oral or genital areas; thus there also was no viral concordance between partners. Risk of infection in the oral cavity of male partners was associated with increasing number of female partners: $\mathrm{OR}=1.1,95 \%$ C.I $=1.0-1.1, p=0.04$ (data not shown). Of the positive oral specimens in men, 1 was HPV-13, and 3 were high-risk types: HPV-31 (2) and HPV-39 (1).

\section{DISCUSSION}

This is one of the first studies of pregnant women to examine concordance in HPV types from multiple cervical specimens and between cervical and oral sites from specimens which were collected concurrently. The lack of concordance in viral types in either of these mucosal sites in pregnant women and between the detection in cervical and oral contact between female and male 
partners suggests that transmission of infection by auto-inoculation or by oral-genital sex between partners is low. Alternatively, our findings may indicate that clearance of HPV from one mucosal site occurs independent of clearance from another site or at different intervals. It remains to be determined whether site-specific tropism of HPV DNA strains can explain the presence of the virus in one site but not another. Our findings do support studies that suggest that the same HPV types reported in the genitals are also found in the oral mucosa.

Because of the timing and location of the recruitment of pregnant women, we were unable to collect specimens from current male partners with which to determine genital HPV status and types. Nonetheless, it is surprising that among the infected males, none of their partners was infected in either the oral or the cervical mucosa. The lack of apparent concordance between partners in our study does not preclude the possibility that males were infected in their genitals and that this was the reservoir of infection for HPV in their partners. Although this source of HPV seems the most plausible explanation for the lack of concordance between partners, this premise is not supported by available data. Koutsky and colleagues recently found that detection of oral HPV was rare in their young adult population and was not associated with oral-penile contact between partners $^{18}$. They did not report on type-specific concordance. Kellokoski et al. ${ }^{19}$ reported the HPV status of 33 current male partners in a cohort of young women. Although the source of HPV infection in the female genitals was thought to be the current partner, there was no concordance in viral types between the oral and anogenital sites of male partners and the oral and cervical specimens in female partners. Hippelainen et al. ${ }^{20}$ also found that HPV detected in the genitals of couples was seldom identical, with only $5.6 \%$ agreement in type. These reports support a hypothesis that the source of currently detected infection may be from an earlier sexual exposure or may be due to infection which has cleared in the other partner or at the other mucosal site.

Another explanation for the lack of concordance between the oral and genital sites within the pregnant women may be due to non- persistent or intermittent detection of HPV over time, as studies have shown in the cervix ${ }^{21-25}$. HPV positivity in the cervix has been shown to increase with multiple sampling over time ${ }^{22,24}$ whereas this phenomenon has not been studied in the oral cavity. Oral and genital specimens may need to be collected at multiple intervals to detect fluctuating levels of infection in an individual, although it is questionable whether repeat measurements would reduce differences in prevalence rates in the two mucosal sites of individuals.

It is unclear whether there are attributes of the oral mucosa that may protect it from the same detectable level or viral infectiosity as in the female genital region. Hormones, such as longterm oral contraceptive use $\mathrm{s}^{26,27}$, have been associated with increased risk of viral detection and cervical SIL, and cancer. Other research supports a hormonal link between number of pregnancies and current pregnancy status associated with a higher prevalence of viral detection and risk of genital cancer ${ }^{7,28,29}$. However, neither oral contraceptive use nor parity in this study showed a clear pattern of risk for HPV detection. Thus it is likely that the mechanism of risk or detectability is associated with other factors. Oral saliva has been shown to be protective against infections through the existence of a number of oral antimicrobial agents such as lysozyme, lactoferrin, $\operatorname{sIgA}$ and cytokines ${ }^{30-32}$. In addition, the highly keratinaized tissue that characterizes the oral cavity may provide a barrier against HPV infection, making it less likely that the virus will invade and reach the basal cell layer than in the cervical mucosa.

Our investigation showed no significant change in the rate of genital HPV DNA prevalence between the third trimester and delivery. This outcome supports some findings ${ }^{6,33,34}$ but is in contrast to investigations which found either a rise between the first and second or third trimesters ${ }^{6,7}$ or a drop in rate after 34 weeks at the time of delivery or postpartum ${ }^{35}$. The hypothesis of a higher rate during the later trimesters is consistent with the increasing rise in the progesterone level as pregnancy progresses. Progesterone has been shown to enhance the transcription and replication of genital HPV 
through the glucocorticoid/progesterone response element found in the long control region of the viral genome ${ }^{36,37}$. Because we did not focus on women in their first trimester of pregnancy, we cannot establish whether their prevalence rate increased between early gestation and later in pregnancy when there were higher levels of progesterone.

A drop in the detection of HPV at the time of delivery is an issue of importance since it may indicate the level of risk from vertical transmission before a vaginal delivery, when the newborn is considered at greatest risk of infection. Several studies $^{38,39}$ including ours ${ }^{33}$ have shown reduced immune responses in pregnant women, suggesting that hormonal effects allow increased replication of infection. We found reduced humoral response to HPV-16 among pregnant compared with non-pregnant women, which dropped progressively by trimester and increased after delivery. A study of local anti-HPV antibodies in cytologically normal women indicated that during late pregnancy ${ }^{39}$ partial suppression of local immunity may have accounted for the relatively poor antibody responses compared to postpartum. That led to a significant rise in the anti-HPV IgA positivity against HPV antigens E2, E7 and L1. De Rosa Husman et al. ${ }^{40}$ found an age-related pattern which may explain in part why study findings differ in regard to positivity rate by trimester. Women aged 15-29 years had a higher prevalence of HPV regardless of trimester compared with women over 30 years of age, but only a slightly higher rate associated with gestational age. Although we found an increased risk of HPV detection in patients $\leqslant 24$ years old who had second-trimester samples compared with those $>30$ years old, the risk was similar $(\mathrm{OR}=2.9,0.7-13.1)$ when comparable age groups were evaluated in third-trimester women $(\mathrm{OR}=3.4,2.0-5.8)$.

One of the strengths of this study was the large number of women from whom both genital and oral specimens were obtained, so that concordance between these two sites could be more adequately addressed. Another advantage was our specimen collection and laboratory procedures which reduce the potential for false-negative results. Great caution is taken to incorporate an adequate and comparable number of nucleated squamous cells in each PCR reaction regardless of the original number of cells collected. Our procedures significantly reduced false-negative detection due to insufficient specimen collection. Only $0.5 \%$ of women had to be eliminated from the study because of an inadequate cell count. This was the first study to use sequencing to assess genital and oral HPV concordance, which further reduced the likelihood of false positive by identifying the specific type and sequence match to known HPV types. This also diminished the reporting of unknown types which may either not represent an HPV infection or may be due to contamination effects. Further, discordance in type within individuals was not resolved by independent testing of samples with PCR amplification with type-specific primers directed at the E6 open reading frame of HPVs. This suggested that the lack of detection was not due to an inability of general PCR to detect multiple HPV types in these cases.

The apparent lack of association between HPV types in the cervix and oral cavity of women and between current partners in our study indicates that a number of issues remain unclear about transmission routes and infectivity of this virus. Other published investigations evaluating infection at these two sites have shown little concordance between sex partners as well ${ }^{24,25}$. Retrieving oral and genital specimens from both partners concurrently is likely to be difficult other than in populations seeking treatment for an abnormal Pap smear or a sexually transmitted disease, as in the populations of both of these previous oral-genital investigations. Such groups are likely to be biased samples and may not reflect the general population prevalence and concordance rates. Most studies evaluating concordance have focused exclusively on genital infection between heterosexual partners who also had either HPV infection or an HPV-related disease rather than on healthy couples ${ }^{41-45}$. The outcome of these investigations is one of mixed results in the degree of concordance between partners. More information about the biological behavior of the virus at different anatomical sites is needed better to understand these findings and the mechanisms of transmission. 


\section{ACKNOWLEDGEMENTS}

This study was supported by grants from NIH NIDCR R01 DE11979 (EMS, JR, DW, THH,
LPT) and Veterans Affairs Merit Review Funds (LPT, THH).

\section{REFERENCES}

1. Walboomer JM, Jacobs MV, Manos MM, et al. Human papillomavirus is a necessary cause of invasive cervical cancer worldwide. J Pathol 1999;189:12-19

2. Ho GY, Burk RD, Klein S, et al. Persistent genital human papillomavirus infection as a risk factor for persistent cervical dysplasia. J Natl Cancer Inst 1995;87:1365-71

3. Hildesheim A, Schiffman MH, Gravitt PE, et al. Persistence of type-specific human papillomavirus infection among cytologically normal women. J Infect Dis 1994;169:235-40

4. Smith EM, Johnson SR, Cripe Pignatari S, Turek LP. Perinatal vertical transmission of human papillomavirus and subsequent development of respiratory tract papillomatosis. Ann Oto Rhinol Laryngo 1991;100:479-83

5. Smith EM, Johnson SR, Cripe T, et al. Perinatal transmission and maternal risks of human papillomavirus infection. Cancer Detect Prev 1995;19: 196-205

6. Watts DH, Koutsky LA, Holmes KK, et al. Low risk of perinatal transmission of human papillomavirus: results from a prospective cohort study. Am J Obstet Gynecol 1998;178:365-73

7. Schneider A, Hotz M, Gissmann L. Increased prevalence of human papillomaviruses in the lower genital tract of pregnant women. Int J Cancer 1987;40:198-201

8. Smith EM, Hoffman HT, Summersgill KS, Kirchner HL, Turek LP, Haugen TH. Human papillomavirus and risk of oral cancer. Laryngoscope 1998;108:1098-103

9. Gillison ML, Koch WM, Capone RB, et al. Evidence for a causal association between human papillomavirus and a subset of head and neck cancers. J Natl Cancer Inst 2000;92: 709-20

10. Kelloski JK, Syrjanen SM, Chang F, Yliskoski M, Syrjanen KJ. Southern blot hybridization and PCR in detection of oral human papillomavirus (HPV) infections in women with genital HPV infections. J Oral Pathol Med 1992;21:1-6
11. National Cancer Institute Workshop. The 1988 Bethesda System for reporting cervical/vaginal cytological diagnoses. JAMA 1989;262:931-4

12. Summersgill KS, Smith EM, Kirchner HL, Haugen TH, Turek LP. p53 polymorphism, human papillomavirus infection, and oral cancer. Oral Surg Oral Med Oral Pathol Oral Radiol Endod 2000;90:334-9

13. Chehab FF, Doherty M, Cai SP, Kan YW, Cooper S, Rubin EM. Detection of sickle cell anaemia and thalassaemias (letter). Nature 1987;329:293-4

14. Gravitt PE, Manos MM. Polymerase chain reaction-based methods for the detection of human papillomavirus DNA. IARC Sci Publ 1992;119:121-33

15. de Roda Husman A-M, Walboomers JM, van den Brule AJ, Meijers CL, Snijers PJ. The use of general primers GP5 and GP6 elongated at their 3' ends with adjacent highly conserved sequences improves human papillomavirus detection by PCR. J Gen Virol 1995;76:1057-62

16. Altschul SF, Madden TL, Schaeffer AA, et al. Gapped BLAST and PSI-BLAST: a new generation of protein database search programs. Nucleic Acids Res 1997;25:3389-402

17. SAS System for Windows. Version 8.2. Cary, NC: SAS Institute, 2001

18. Winer RL, Lee S-K, Hughes JP, Adam DE, Kiviat NB, Koutsky LA. Genital human papillomavirus infection: incidence and risk factors in a cohort of female university students. $A m$ J Epidemiol 2003;157:218-26

19. Kellokoski JK, Syrjanen S, Yliskoski M, Syrjanen K. Dot blot hybridization in detection of human papillomavirus (HPV) infections in the oral cavity of women with genital HPV infections. Oral Microbiol Immunol 1992;7:19-23

20. Hippelainen MI, Yliskoski M, Syrjanen S, et al. Low concordance of genital human papillomavirus (HPV) lesions and viral types in HPVinfected women and their male sexual partners. Sex Trans Dis 1994;21:76-82 
21. Brisson J, Bairati I, Morin C, et al. Determinants of persistent detection of human papillomavirus DNA in the uterine cervix. J Infect Dis 1996;173: 794-99

22. Wallin K-L, Wiklund F, Angstrom T, et al. Typespecific persistence of human papillomavirus DNA before the development of invasive cervical cancer. N Engl J Med 1999;341:1633-8

23. Wheeler CM, Greer CE, Becker TM, Hunt WC, Anderson SM, Manos MM. Short-term fluctuations in the detection of cervical human papillomavirus DNA. Obstet Gynecol 1996;88: 261-8

24. Schneider A, Kirschhoff T, Meinhardt G, Gissmann L. Repeated evaluation of human papillomavirus 16 status in cervical swabs of young women with a history of normal papanicolaou smears. Obstet Gynecol 1992;79:683-8

25. Moscicki AB, Palefsky J, Smith G, Soboshski S, Schoolnik G. Variability of human papillomavirus DNA testing in a longitudinal cohort of young women. Obstet Gynecol 1993;82:578-85

26. Ylitalo N, Sorensen P, Josefsson A, et al. Smoking and oral contraceptives as risk factors for cervical carcinoma in situ. Int J Cancer 1999;81:357-65

27. Lacey Jr JV, Brinton LA, Abbas FM, et al. Oral contraceptives as risk factors for cervical adenocarcinomas and squamous cell carcinomas. Cancer Epidemiol Biomarkers Prev 1999;8:1079-85

28. Rando RF, Lindheim S, Hasty L, Sedlacek TV, Woodland M, Eder C. Increased frequency of detection of human papillomavirus deoxyribonucleic acid in exfoliated cervical cells during pregnancy. Am J Obstet Gynecol 1989;161:50-5

29. Morrison EA, Gammon MD, Goldberg GL, Vermund SH, Burk RD. Pregnancy and cervical infection with human papillomaviruses. Int J Gynaecol Obstet 1996;54:125-30

30. Shugars DC, Watkins CA, Cowen HJ. Salivary concentration of secretory leukocyte protease inhibitor, an antimicrobial protein, is decreased within advanced age. Gerontology 2001;47:246-53

31. Miletic ID, Schiffman SS, Miletic VD, SatterlyMiller EA. Salivary IgA secretion rate in young and elderly persons. Physiop Behav 1996;60:243-8

32. Steele C, Fidel Jr PL. Cytokine and chemokine production by human oral and vaginal epithelial cells in response to Candida albicans. Infect Immun 2002;70:577-83

33. Chang-Claude J, Schneider A, Smith E, Blettner M, Wahrendorf J, Turek L. Longitudinal study of the effects of pregnancy and other factors on detection of HPV. Gynecol Oncol 1996;60:355-62
34. Kemp EA, Hakenwerth AM, Laurent SL, Everitt PE, Stoerker J. Human papillomavirus prevalence in pregnancy. Obstet Gynecol 1992;79:649-56

35. Smith EM, Johnson SR, Jiang D, et al. The association between pregnancy and human papillomavirus prevalence. Cancer Detect Prev 1991;15: 397-402

36. Chan WK, Klock G, Bernard HU. Progesterone and glucocorticoid response elements occur in the long control regions of several human papillomaviruses involved in anogenital neoplasia. J Virol 1989;63:3261-9

37. Pater A, Bayatpour M, Pater MA. Oncogenic transformation by human papillomavirus type 16 deoxyribonucleic acid in the presence of progesterone or progestins from oral contraceptives. Am J Obstet Gynecol 1990;162: 1099-103

38. Shneh S, Muller M, Schneider A, et al. Serologic response to the E4, E6, and E7 proteins of human papillomavirus type 16 in pregnant women. Am J Obstet Gynecol 1998;178: 360-4

39. Veress G, Csiky-Mészáros T, Kónya J, Czeglédy J, Gergely L. Follow-up of human papillomavirus (HPV) DNA and local anti-HPV antibodies in cytologically normal pregnant women. Med Microbiol Immunol 1996;185:139-44

40. de Rosa Husman AM, Walboomers JM, Hopman $\mathrm{E}$, et al. HPV prevalence in cytomorphologically normal cervical scrapes of pregnant women as determined by PCR: the age-related pattern. J Med Virol 1995;46:97-102

41. Baraggino E, Stinco G, Wiesenfeild U. Study of the partners of women with human papillomavirus infection. Int J Dermatol 1993;32:661-3

42. Barrasso R, DeBrux J, Croissant O, Orth G. High prevalence of papillomavirus-associated penile intraepithelial neoplasia in sexual partners of women with cervical intraepithelial neoplasia. $N$ Engl J Med 1987;317:916-23

43. Bar-Am A, Niv J, Jaffo A, Jaffo A, Peyser RM. Prevalence of human papillomavirus infection and HPV DNA among male partners of Israeli women with genital premalignant and human papillomavirus lesions. Isr J Med Sci 1995;31: 349-52

44. Maymon R, Bekerman A, Werchow M, Maymon BB, Segal R, Faktor JH. Clinical and subclinical condyloma. Rates among male sexual partners of women with genital human papillomavirus infection. $J$ Reprod Med 1995;40: $31-6$ 
45. Wickenden C, Hanna N, Taylor-Robinson D, et al. Sexual transmission of human papillomaviruses in heterosexual and male homosexual couples, studied by DNA hybridization. Genitourinary Medicine 1988;64:34-8

RECEIVED 06-09-03; ACCEPTED 11-03-03 


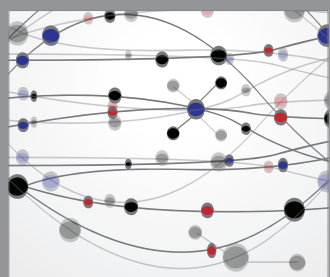

The Scientific World Journal
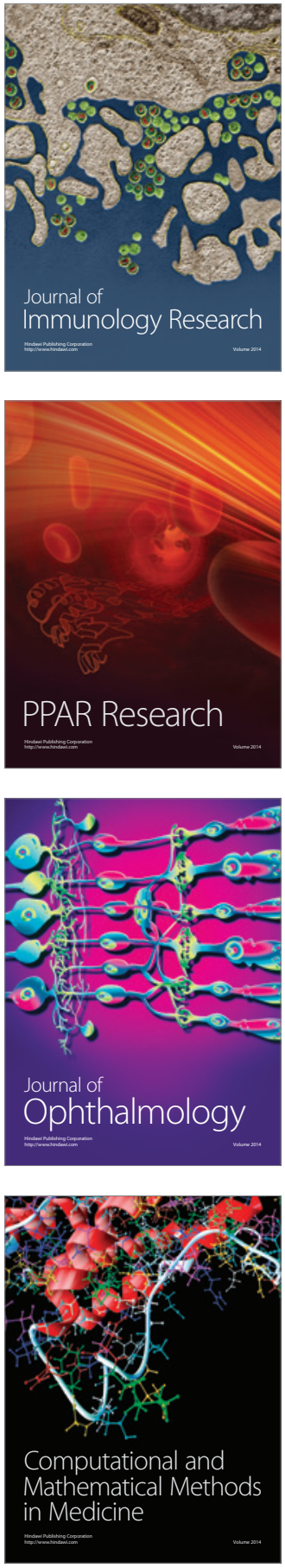

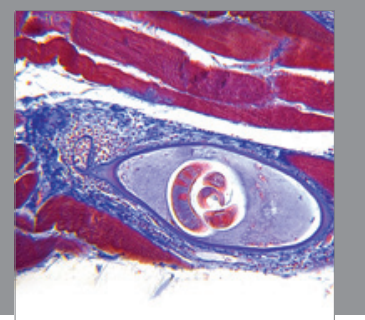

Gastroenterology

Research and Practice
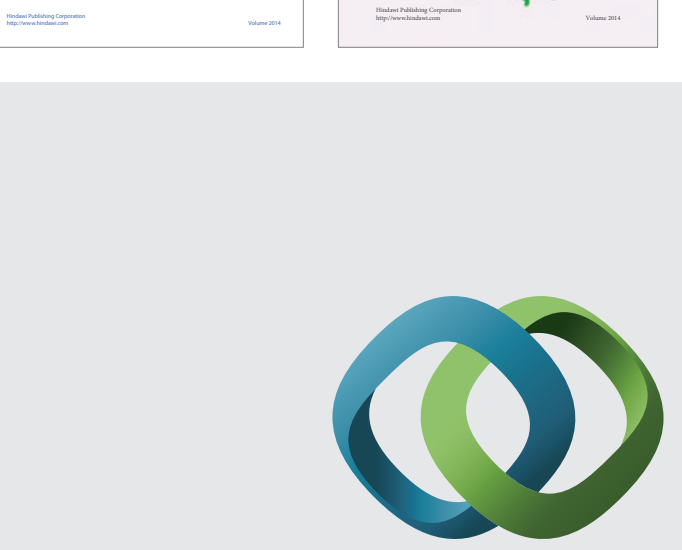

\section{Hindawi}

Submit your manuscripts at

http://www.hindawi.com
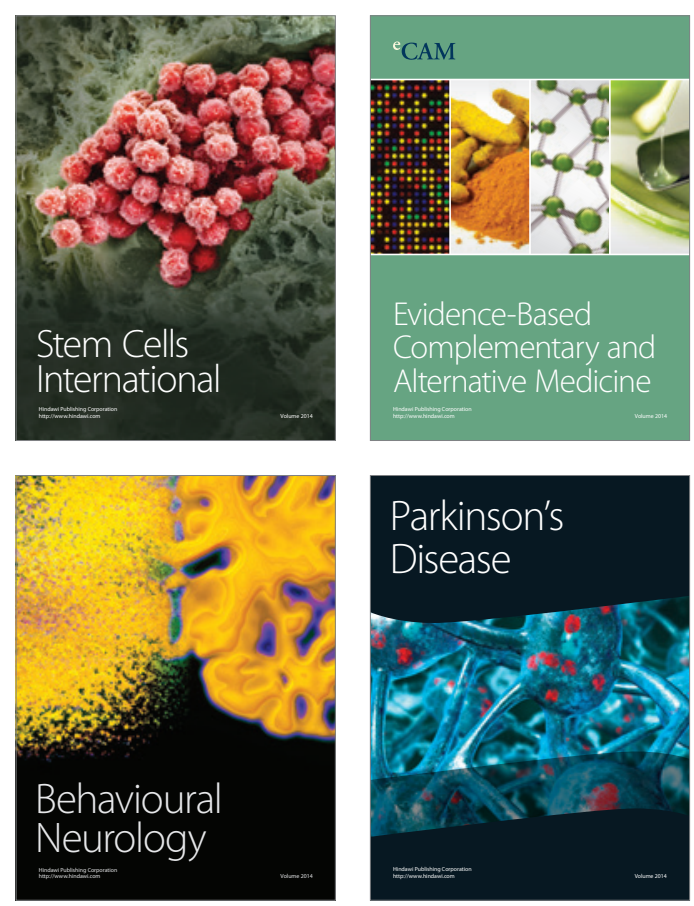

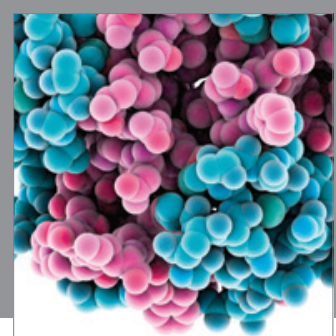

Journal of
Diabetes Research

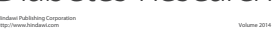

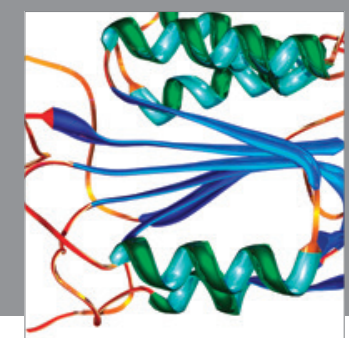

Disease Markers
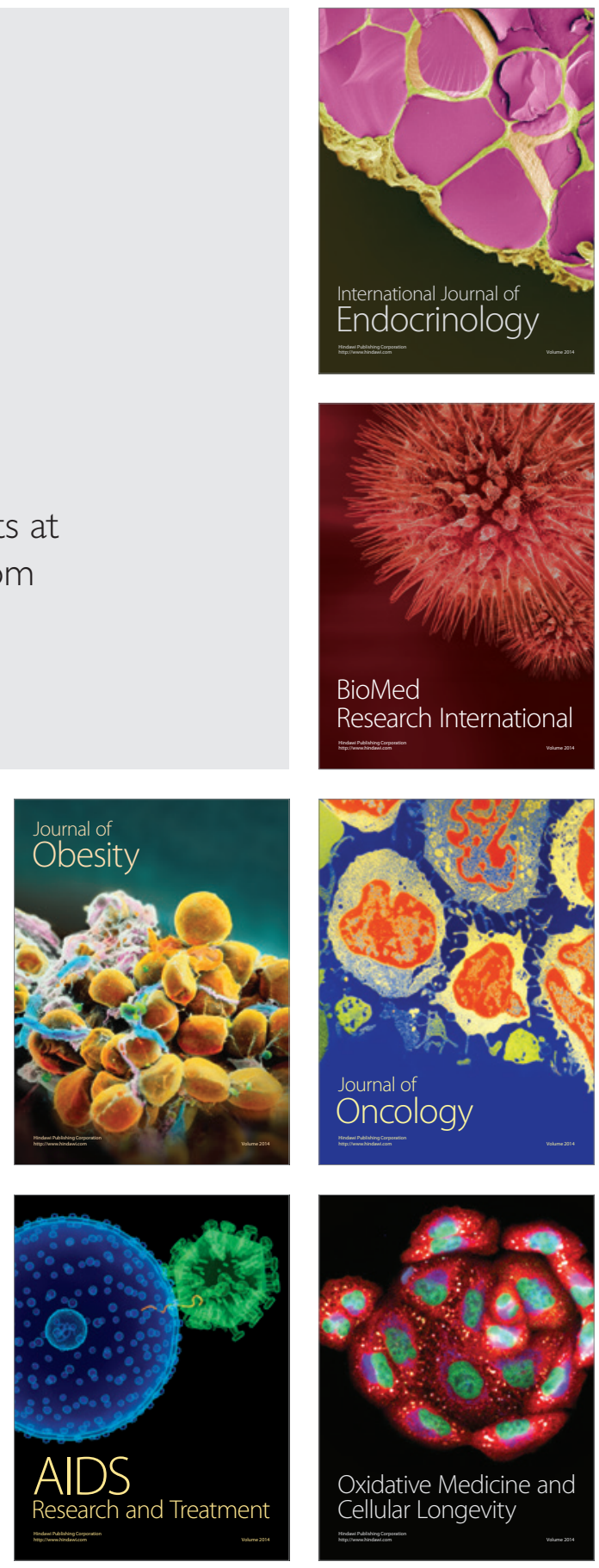